1 F. J. Welcher, Organic Analytical Reagents, Vol. III, Chap. XIII, 1955.

2 J. Stary, The Solvent Extraction of Metal Chelates, Pergamon Press, New York 1964.

3 M. Piskorzand and T. Urbanski, Bull. Acad. Polon. Sci. Ser. Chem. 11, 597 [1963].

${ }^{4}$ N. V. Thakur, V. B. Kastha, C. R. Kanekr, and V. R. Marathe, J. Inorg. Nuclear Chem. 34, 2831 [1972].

5 A. H. Abou El Ela, F. M. Abdel Kerim, H. H. Afifi, and H. F. Aly, Z. Naturforsch. 28 b, [1973].
6 T. Yoshimura, C. Miyake, and S. Imoto, Bull. Chem. Soc. Jap. 45, 5, 1424 [1972].

7 Y. Tanabe and S. J. Sugano, J. Phys. Soc. Jap. 9, 753 [1954].

8 B. N. Figgis, Introduction to Ligand Field Theory, Interscience Publication, New York 1966.

9 C. K. Jorgensen, Acta Chem. Scand. 9, 116 [1955].

10 A. D. Liehr and C. J. Ballhausen, Ann. Phys. (N. Y.), 6, 134 [1959].

11 H. H. Jaffe and M. Orchin, Theory and Applications of UV Spectroscopy, John Wiley \& Sons, New York 1962.

\title{
Polaron Conduction and Optical Absorption of Amorphous Selenium Films
}

\author{
A. H. Abou El Ela \\ Physics Department, National Research Centre, Cairo, U.A.R. \\ (Z. Naturforsch. 29 a, 724-727 [1974] ; received December 19, 1973)

\begin{abstract}
The temperature dependence of electrical conductivity in amorphous selenium films is in vestigated. The experimental results show a polaronic conduction in the states near the Fermi level, in agreement with Mott's model. Optical absorption measurements show the rate at which the valence band states fall off with energy into the energy gap.
\end{abstract}

The mechanism of electronic conduction in amorphous materials is not yet understood. Because of the disordered lattice structure, the energy band structure cannot be as readily derived for these materials as it has been for periodic lattices. Nevertheless, it is believed that valence and conduction bands do exist for the amorphous structure, but a true band gap of forbidden energies between the valence and conduction bands probably does not exist. Instead, energy states at the band edges may smear or tail into a pseudo-gap. Within this pseudogap the states are localized, and outside the pseudogap, in the valence and conduction bands, the hole and electron states are nonlocalized as in the valence and conduction bands of crystalline semiconductors ${ }^{1,2}$. Electronic conductivity could take place by hopping of carriers in the localized states. In order to identify the mechanism of conduction for amor. phous selenium, we have conducted measurements of the temperature dependence of conductivity on various amorphous selenium films.

\section{Electrical Conductivity}

Amorphous selenium films were prepared by thermal evaporation of spectral pure selenium in a vacuum of $\sim 10^{-5}$ torr at an evaporation rate of
$15 \AA$ per second from a tantalum boat onto quartz plates. The samples were provided with silver electrodes. The so-formed contacts are not blocking. Measurements were made for two different voltages applied to the sample: 1500 and 2000 volt using a stabilized high voltage power supply. The current which flowed through the sample was measured by a Vibrating Reed Electrometer sensitive to detect currents as low as $10^{-15}$ A. Figure 1 shows the dependence of the current intensity (plotted on log scale) on the reciprocal temperature at different applied voltages. Sample thickness of $1553 \AA$ and $6550 \AA$ (determined by interferometric methods ${ }^{3}$ were used. The $2000 \mathrm{~V}$ curve shows a change of slope around $240 \mathrm{~K}$. For temperatures above $240 \mathrm{~K}$, the slope of the straight line is independent of the applied voltage and gives an activation energy about $0.43 \mathrm{eV}$, whereas for temperatures below $240 \mathrm{~K}$ the activation energy is strongly influenced by the applied voltage. The presence of more than one activation energy was observed on $\mathrm{As}_{2} \mathrm{Se}_{3}$ samples ${ }^{4}$. Rossiter and Warfield ${ }^{5}$ reported a change in the mobility activation energy with temperature, in amorphous selenium. Several activation energies in the conductivity of amorphous silicon layers were observed ${ }^{6}$ and interpreted as a result of the hopping process. Moreover, the existance of a critical tem- 


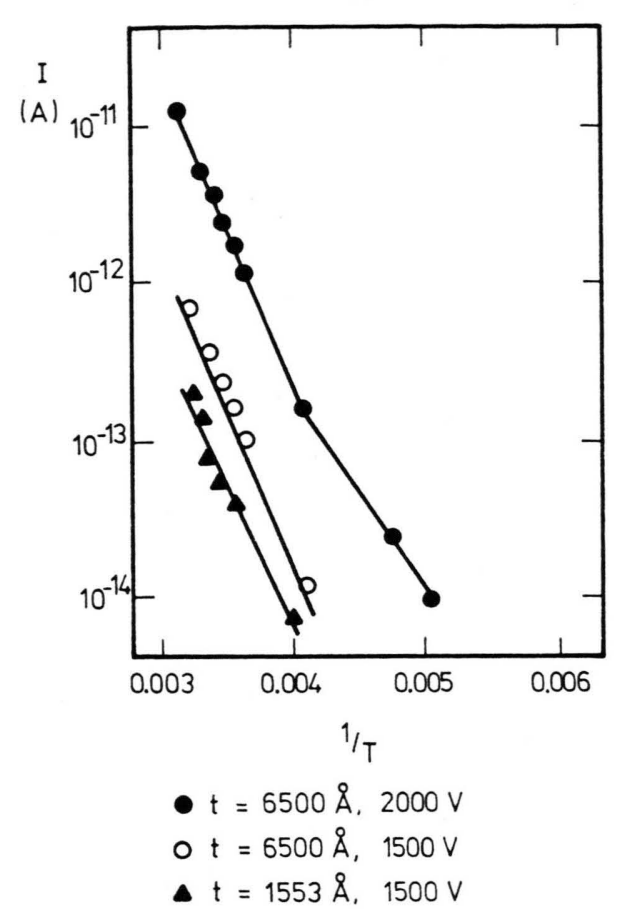

Fig. 1. Current intensity in amorphous selenium films plotted in log scale versus reciprocal temperature.

perature at which the activation energy changes suggests the possibility of polaron conduction ${ }^{7}$. Hempel ${ }^{8}$ has suggested a polaronic conduction for selenium.

Mott ${ }^{9}$ studied the conductivity for energies $E$ near an energy $E_{\mathrm{c}}$, which separates localized and non-localized states, and derived, for the localized states, an expression for the polaron mobility,

$$
\mu(E)=\left(\nu_{\mathrm{p}} a^{2} \Phi e / K T\right) \exp \{-W / K T\}
$$

with $W$ the hopping energy, $v_{\mathrm{p}}$ the phonon energy $\left(10^{13} \mathrm{sec}^{-1}\right)$, and $a$ the distance between atoms. The approximation $v_{\mathrm{p}}=10^{13} \mathrm{sec}^{-1}$ is valid only if $W>K \Phi_{\mathrm{D}}$ with $\Theta_{\mathrm{D}}$ the debye temperature. It is no: valid for impurity conduction at low temperatures. $\Phi$ is a number depending on the overlap between wave functions. It contains the factor $\exp \{-2 \delta R\}$ if the overlap between states is small ( $\delta$ is the rate at which the atomic wave function falls off with distance and $R$ is the mean distance between the states near the Fermi level). If, however, the overlap is considerable this factor will be of order unity. Moreover, as $E \rightarrow E_{\text {c }}$ each localized state will overlap a large number of others and $\Phi$ becomes much larger than unity.
Polarons arise from the interaction between an electric field created by a localized electron and the polarization field of the lattice. For temperatures above $\frac{1}{2} \Theta_{D}$ polarons move by hopping with a mobility given by expression (1). For temperatures below $\frac{1}{2} \Theta_{\mathrm{D}}$, when the conductivity depends on the behaviour of electrons with energies near $E_{\mathrm{F}}$, only those electrons with energies $\sim K T$ below $E_{\mathrm{F}}$ have a significant probability of hopping and $W$ tends to zero.

Mott ${ }^{10}$ found that the conductivity, when hopping is not between nearest neighbiours but between more distant centres, should obey

$$
\ln \sigma=A-B T^{-1 / 4}
$$

where $A$ and $B$ are constants. Figure 2 shows the dependence of the current intensity, plotted in log scale, on $T^{-1 / 4}$. For temperatures below $240 \mathrm{~K}$, the curve is a straight line in full agreement with relation (2). Therefore, one may consider that the conduction arises from polaron transport by a hopping

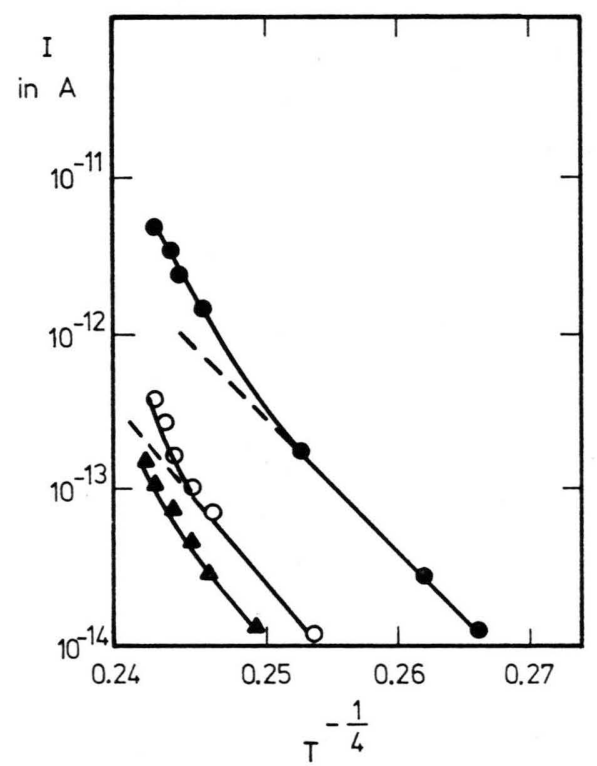

Fig. 2. Current intensity versus $T^{-1 / 4}$ at different voltages.

process in the localized states near the Fermi level.

For temperatures above $\frac{1}{2} \Theta_{\mathrm{D}}(\sim 240 \mathrm{~K})$ the conductivity is related to the temperature ${ }^{11}$ by the relation $\sigma=D T \mu=\sigma_{0} \exp \{-W / K T\}$. The value of $W$ was obtained from the plot of $\log \sigma$ versus $1 / T$ and is equal to $0.43 \mathrm{eV}$.

Inspite of the presence of injected carriers, due to high electric fields applied on the sample, the 
theories of conduction by hopping process and polarons can be used ${ }^{12}$, since the quasi-thermal equilibrium is conserved.

\section{Optical Absorption}

The optical absorption edge of nearly all amorphous materials is characterized by an exponential or Urbach ${ }^{13}$ dependence of the absorption constant $\alpha$ on the photon energy $h v<E_{\mathrm{g}}$ :

$$
\alpha(h v)=\alpha_{0}(T) \exp \left\{\gamma\left[h v-E_{\mathrm{g}}(T) / K T\right\}\right.
$$

$\alpha_{0}$ is the normalized transition strength, $\gamma$ a constant of order unity and $E_{\mathrm{g}}$ the energy gap. Urbach behaviour is observed in some crystalline materials, however a unique explanation for the effect has not yet been found.

For the amorphous phase, because of the small spatial overlap between those states in the valence and conduction bands which are localized, optically induced transitions between these occur with low probability. However, transitions from the valence

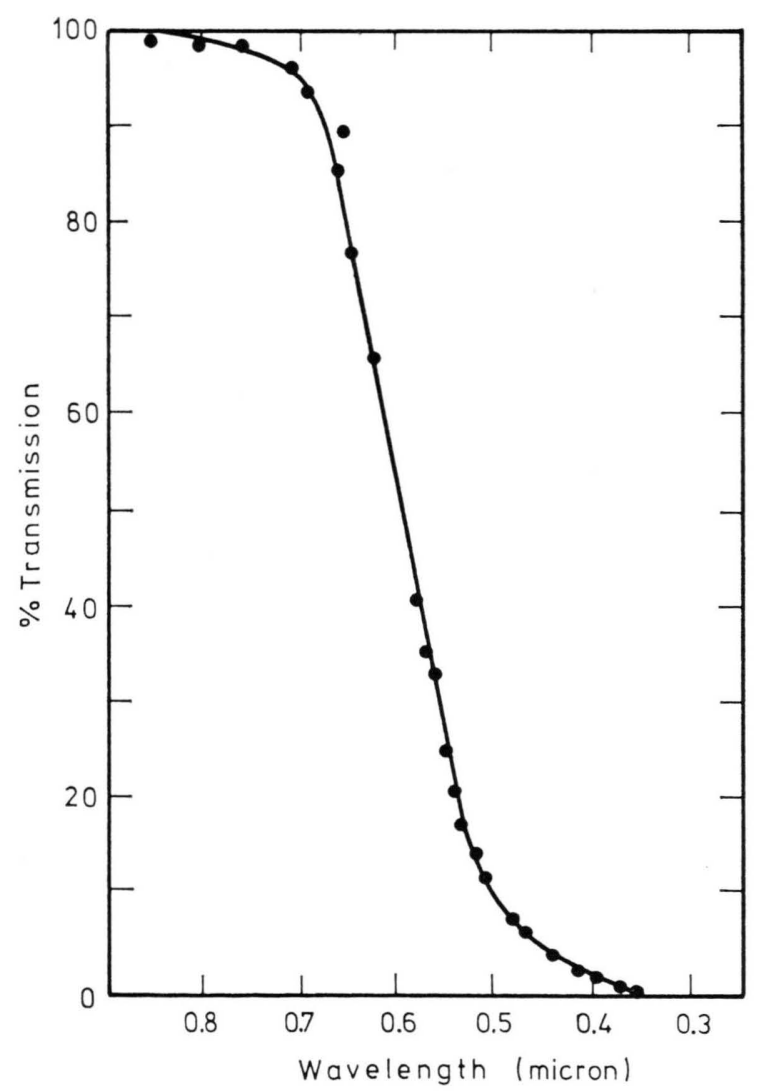

Fig. 3. The dependence of sample transmission on the wavelength. band tail to nonlocalized states in the conduction band should contribute appreciably to the absorption. Simple calculations ${ }^{14,15}$ show that the absorption coefficient as a function of photon energy should reflect the rate at which the valence band states fall off with energy into the gap.

Optical transmission measurements were carried out on amorphous selenium films, using a Beckman UV spectrophotometer D.U. Figure 3 shows the variation of sample transmission with the wavelength for film thickness $1553 \AA$. The transmission falls off rapidly for wavelengths lower than $7500 \AA$. The variation of the absorption coefficient, calculated from transmission data, with incident photon energy $h v$ is shown in Figure 4. The plot of the

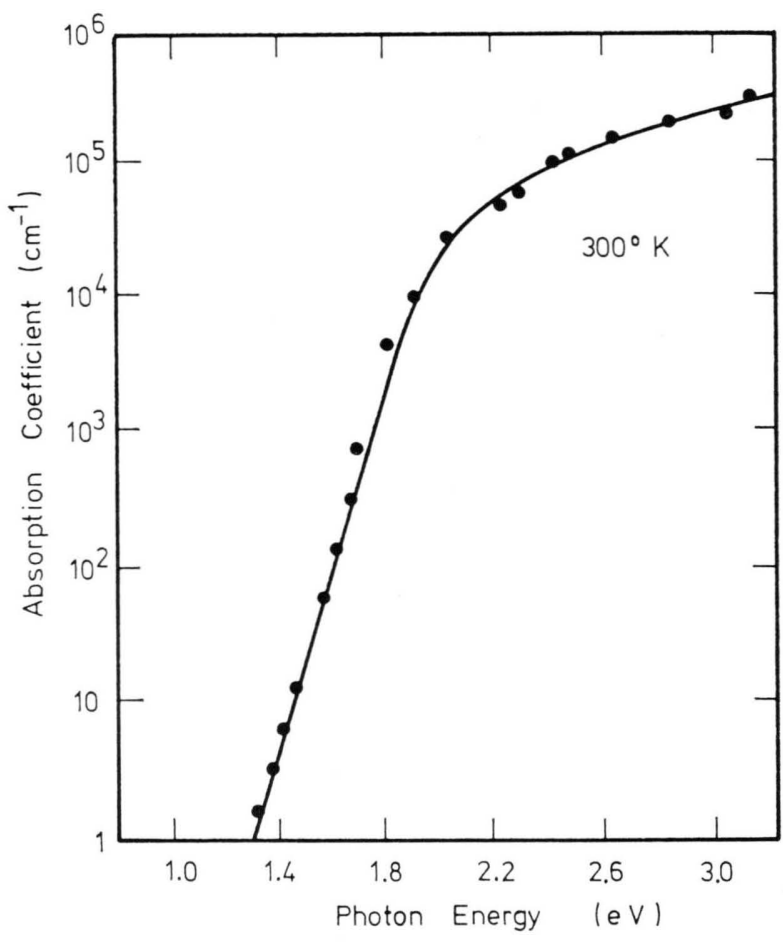

Fig. 4. Optical absorption coefficient versus photon energy.

logarithm of the absorption coefficient against $h v$ is a straight line in the region characterizing the Urbach behaviour.

At high values of the optical absorption coefficient $\left(10^{4}-10^{6} \mathrm{~cm}^{-1}\right)$ deviations from the Urbach behaviour occur in selenium. These, it is believed, occur at photon energies corresponding to excitations final states beyond the band tail (where the 
density of states are large). A reasonable fit to the curve in this region has been obtained using the conventional formula for semiconductors with parabolic bands

1 N. F. Mott, Adv. Phys. 16, 49 [1967].

2 M. H. Cohen, H. Fritzche, and S. R. Ovshinsky, Bull. Amer. Phys. Soc. 14, 311 [1969].

3 S. Tolansky, Multiple Beam Interferometry, England.

4 A. E. Owen and J. M. Robertson, J. Non-Cryst. Sol. 2, 40 [1970].

5 E. L. Rossiter and G. Warfield, Dept. Elect. Eng. Device, Phys. Lab. Princeton University, Tech. Report No 10 [1967].

6 P. G. Le Comber and W. E. Spear, Phys. Rev. Letters 25, 509 [1970].

7 I. G. Austin and N. F. Mott, Adv. Phys. 18, 4l [1969].

$$
\alpha \sim\left[\frac{h v-E_{\mathrm{g}}}{h v}\right]^{n}
$$

with a value of $n=2$, which is close to the value suggested by Mott.

${ }^{8}$ H. P. Hempel, Recent Advances in Selenium Physics, Pergamon Press, London 1965, p. 109.

9 N. F. Mott, Phil. Mag. 19, 835 [1969].

10 N. F. Mott, J. Non-Cryst. Sol. 1, 1 [1968].

11 N. F. Mott, Festkörperprobleme 9, 22 [1969].

12 C. Vautier, D. Carles, and C. Vigar, J. Non-Cryst. Sol. 7, 117 [1972].

13 F. Urbach, Phys. Rev. 92, 1324 [1953].

14 H. P. D. Lanyon, Phys. Rev. 130, 134 [1961].

15 J. Tauc, Optical Properties of Solids, Ed. F. Abeles, NorthHolland, Amsterdam 1969. 